\title{
Tight-Binding Modeling of Uranium in Aqueous Environment
}

Rebecca K. Carlson, Marc J. Cawkwell, Enrique R. Batista, and Ping Yang

Theoretical Division, Los Alamos National Laboratory, Los Alamos, New Mexico, 57545, United States

\section{Table of Contents}

DFTB parameters

p. 2

List of Structures in Training Set

p. 3

Figures of Structures in Training Set

p. 3-5

List of Molecules and structures for Data in Table 1

p. 6-7

Tables comparing CASPT2, MN15, DFTB geometries for small molecules

p. 8

Tables of energetic results

p. $9-12$

Dimer Structures

p.13-15 


\section{DFTB parameters}

Table S1. Onsite Energies (eV) for $\mathrm{U}, \mathrm{O}$, and $\mathrm{H}$

\begin{tabular}{|c|c|c|c|c|}
\hline & $\varepsilon_{S}$ & $\varepsilon_{p}$ & $\varepsilon_{d}$ & $\varepsilon_{f}$ \\
\hline $\mathrm{U}$ & -4.080 & & 0.816 & -0.586 \\
\hline $\mathrm{O}$ & -23.770 & -8.850 & & \\
\hline $\mathrm{H}$ & -6.240 & & & \\
\hline
\end{tabular}

Table S2. Optimized Parameters for Pair Potentials between $\mathrm{U}, \mathrm{O}$, and $\mathrm{H}$

$$
\begin{array}{lllllll}
\Phi_{0}(\mathrm{eV}) & A_{1}\left(\AA^{-1}\right) & A_{2}\left(\AA^{-1}\right) & A_{3}\left(\AA^{-1}\right) & A_{4}\left(\AA^{-1}\right) & R_{1}(\AA) & R_{\text {cut }}(\AA)
\end{array}
$$

$\begin{array}{llllllll}\text { U-U } & 13.010148 & -3.196768 & -0.222285 & -1.535983 & 1.700000 & 3.0 & 3.5\end{array}$

$\begin{array}{llllllll}\text { U-O } & 1.110490 & -10.469888 & -15.488347 & -22.002678 & 1.200000 & 2.0 & 2.5\end{array}$

$\begin{array}{llllllll}\text { U-H } & 2.018322 & -4.612853 & -0.311508 & -5.875073 & 1.000000 & 1.6 & 2.0\end{array}$

$\begin{array}{llllllll}\mathrm{O}-\mathrm{O} & 0.220246 & -17.301035 & -27.408741 & 0.000000 & 0.750000 & 1.2 & 1.4\end{array}$

$\begin{array}{llllllll}\mathrm{O}-\mathrm{H} & 1.623788 & -4.826154 & -8.080753 & -12.112240 & 2.000000 & 3.0 & 3.5\end{array}$

$\begin{array}{llllllll}\mathrm{H}-\mathrm{H} & 3.646270 & -2.287960 & 1.343740 & -0.343381 & 2.800000 & 5.0 & 6.0\end{array}$

Table S3. Hubbard $U(\mathrm{eV})$ and Mulliken Spin parameters $W$, for $\mathrm{U}, \mathrm{O}$, and $\mathrm{H}$

\begin{tabular}{|c|c|c|c|c|}
\hline & $U$ & $W_{s}$ & $W_{p}$ & $W_{f}$ \\
\hline $\mathrm{U}$ & 9.0 & & & -0.355 \\
\hline $\mathrm{O}$ & 12.2 & & -0.758 & \\
\hline $\mathrm{H}$ & 12.8 & -2.234 & & \\
\hline
\end{tabular}


Table S4. Number of Distortions in Training Set, overall spin state of molecules, and formal oxidation state of $U$

\begin{tabular}{|c|c|c|c|}
\hline & $\begin{array}{l}\text { \# distortions } \\
\text { Training set }\end{array}$ & Spin state & Formal Oxidation State \\
\hline $\mathrm{UH}_{3}$ & 364 & quartet & $\mathrm{U}(\mathrm{III})$ \\
\hline $\mathrm{UH}_{4}$ & 39 & triplet & U(IV) \\
\hline $\mathrm{UO}_{2} \mathrm{H}$ & 26 & doublet & $\mathrm{U}(\mathrm{V})$ \\
\hline $\mathrm{UH}_{3} \mathrm{O}$ & 80 & doublet & $\mathrm{U}(\mathrm{V})$ \\
\hline $\mathrm{UH}_{2} \mathrm{O}$ & 233 & triplet & U(IV) \\
\hline Total U-H & 742 & & \\
\hline $\mathrm{UO}_{2} \mathrm{H}$ & 26 & doublet & $\mathrm{U}(\mathrm{V})$ \\
\hline $\mathrm{UH}_{3} \mathrm{O}$ & 80 & doublet & $\mathrm{U}(\mathrm{V})$ \\
\hline $\mathrm{UH}_{2} \mathrm{O}$ & 233 & triplet & U(IV) \\
\hline $\mathrm{UO}_{2}^{2+}$ & 678 & singlet & U(VI) \\
\hline $\mathrm{UO}_{2}\left(\mathrm{H}_{2} \mathrm{O}\right)^{2+}$ & 396 & singlet & $\mathrm{U}(\mathrm{VI})$ \\
\hline $\mathrm{UO}_{2}\left(2 \mathrm{H}_{2} \mathrm{O}\right)^{2+}$ & 404 & singlet & U(VI) \\
\hline $\mathrm{UO}_{2}\left(3 \mathrm{H}_{2} \mathrm{O}\right)^{2+}$ & 39 & singlet & U(VI) \\
\hline $\mathrm{UO}_{2}\left(4 \mathrm{H}_{2} \mathrm{O}\right)^{2+}$ & 62 & singlet & U(VI) \\
\hline $\mathrm{UO}_{2}\left(5 \mathrm{H}_{2} \mathrm{O}\right)^{2+}$ & 6 & singlet & U(VI) \\
\hline $\mathrm{UO}_{2}(\mathrm{OH})^{+}$ & 453 & singlet & U(VI) \\
\hline $\mathrm{UO}_{2}(\mathrm{OH})\left(\mathrm{H}_{2} \mathrm{O}\right)^{+}$ & 226 & singlet & U(VI) \\
\hline $\mathrm{UO}_{2}(\mathrm{OH})\left(\mathrm{H}_{2} \mathrm{O}\right)_{2}^{+}$ & 38 & singlet & U(VI) \\
\hline $\mathrm{UO}_{2}(\mathrm{OH})\left(\mathrm{H}_{2} \mathrm{O}\right)_{3}{ }^{+}$ & 17 & singlet & $\mathrm{U}(\mathrm{VI})$ \\
\hline $\mathrm{UO}_{2}(\mathrm{OH})_{2}$ & 233 & singlet & U(VI) \\
\hline $\mathrm{UO}_{2}(\mathrm{OH})_{2}\left(\mathrm{H}_{2} \mathrm{O}\right)$ & 165 & singlet & U(VI) \\
\hline $\mathrm{UO}_{2}(\mathrm{OH})_{2}\left(\mathrm{H}_{2} \mathrm{O}\right)_{2} \mathrm{cis}$ & 79 & singlet & U(VI) \\
\hline $\mathrm{UO}_{2}(\mathrm{OH})_{2}\left(\mathrm{H}_{2} \mathrm{O}\right)_{2}$ trans & 57 & singlet & U(VI) \\
\hline $\mathrm{U}(\mathrm{OH})_{4}$ & 142 & triplet & U(IV) \\
\hline $\mathrm{U}(\mathrm{OH})_{4}\left(\mathrm{H}_{2} \mathrm{O}\right)_{2}$ & 37 & triplet & U(IV) \\
\hline $\mathrm{UO}(\mathrm{OH}) 5^{-}$ & 220 & singlet & $\mathrm{U}(\mathrm{VI})$ \\
\hline Total U-O & 3591 & & \\
\hline
\end{tabular}

Structures in training set listed in Table S4. Solid wedges indicate a bond coming out of page and dashed wedge indicates bond going back into page.

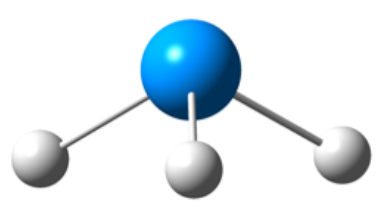

$\mathrm{UH}_{3}$

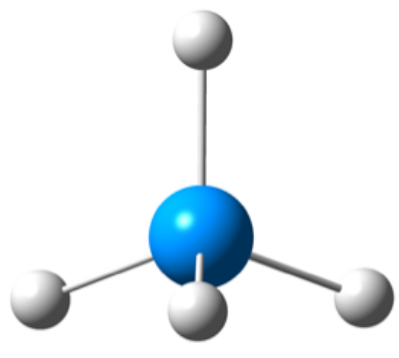

$\mathrm{UH}_{4}$

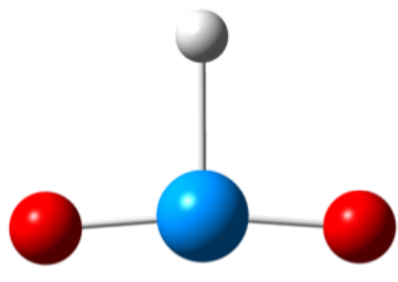

$\mathrm{UO}_{2} \mathrm{H}$ 


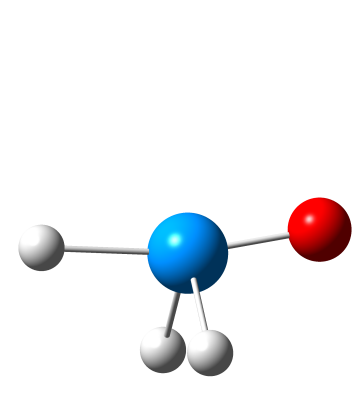

$\mathrm{UH}_{3} \mathrm{O}$
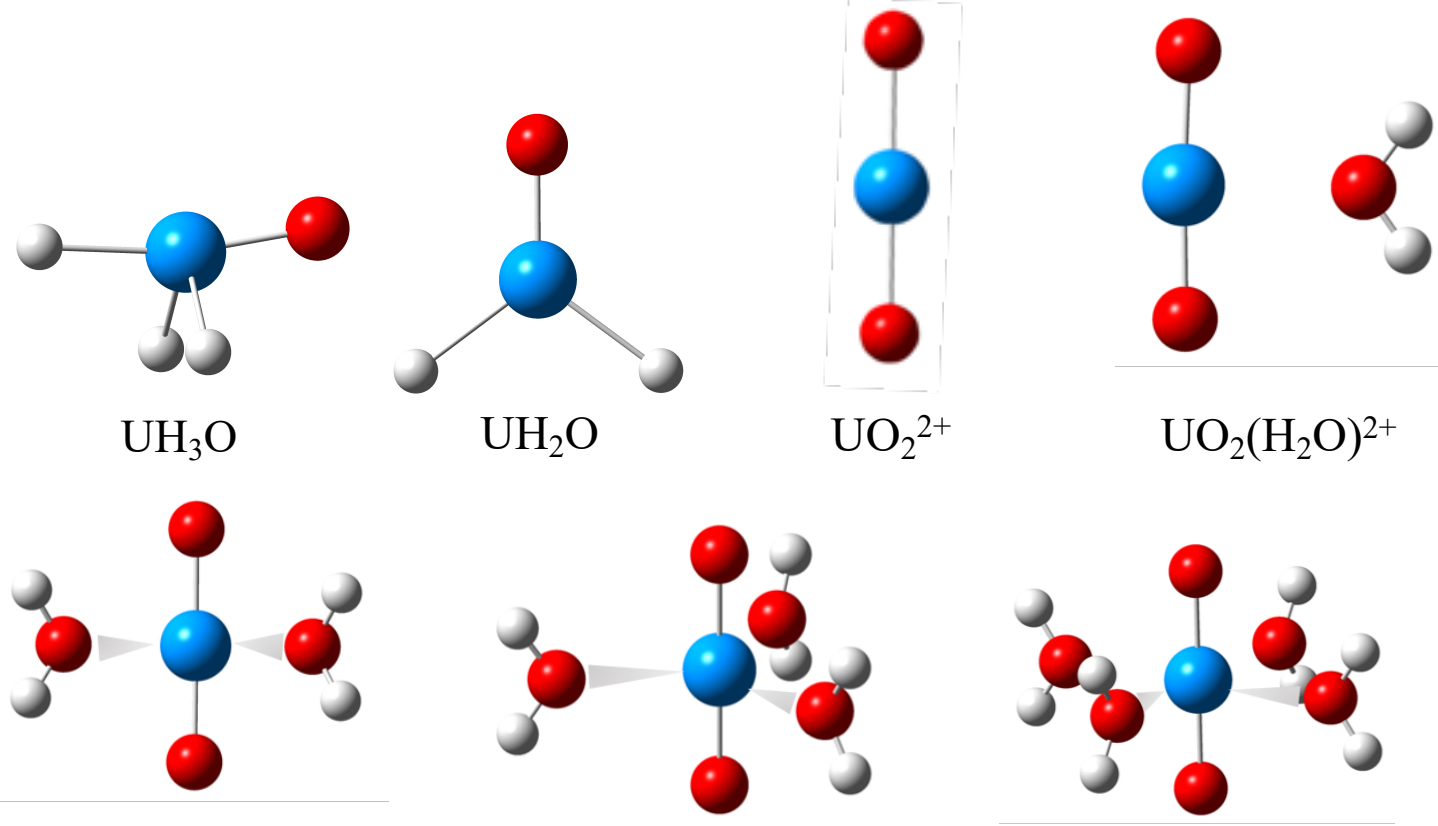

$\mathrm{UO}_{2}\left(\mathrm{H}_{2} \mathrm{O}\right)_{2}{ }^{2+}$

$\mathrm{UO}_{2}\left(\mathrm{H}_{2} \mathrm{O}\right)_{3}{ }^{2+}$

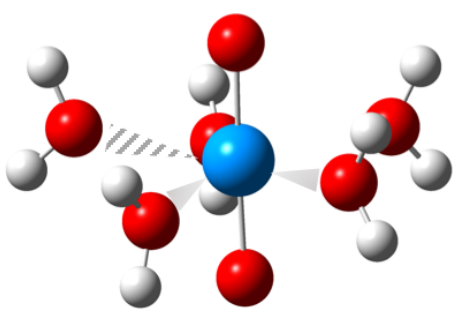

$\mathrm{UO}_{2}\left(\mathrm{H}_{2} \mathrm{O}\right)_{5}{ }^{2+}$

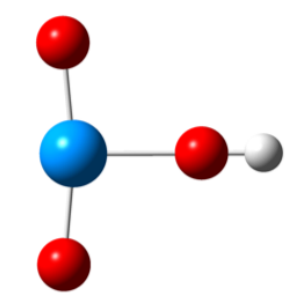

$\mathrm{UO}_{2}(\mathrm{OH})^{+}$

$\mathrm{UO}_{2}{ }^{2+}$

$\mathrm{UO}_{2}\left(\mathrm{H}_{2} \mathrm{O}\right)^{2+}$

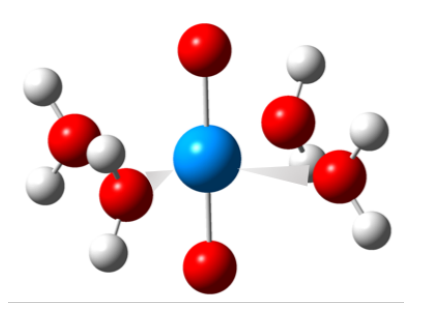

$\mathrm{UO}_{2}\left(\mathrm{H}_{2} \mathrm{O}\right)_{4}{ }^{2+}$

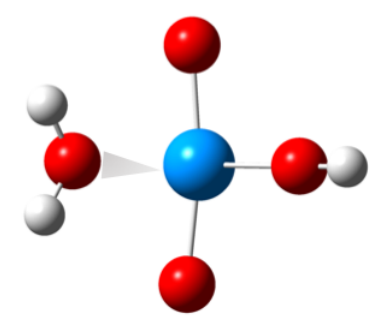

$\mathrm{UO}_{2}(\mathrm{OH})\left(\mathrm{H}_{2} \mathrm{O}\right)^{+}$

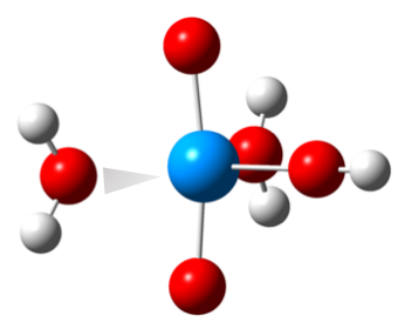

$\mathrm{UO}_{2}(\mathrm{OH})\left(\mathrm{H}_{2} \mathrm{O}\right)_{2}{ }^{+}$ 


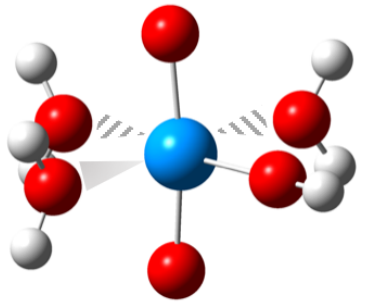

$\mathrm{UO}_{2}(\mathrm{OH})\left(\mathrm{H}_{2} \mathrm{O}\right)_{3}{ }^{+}$

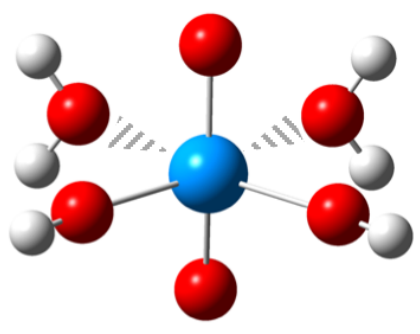

$\mathrm{UO}_{2}(\mathrm{OH})_{2}\left(\mathrm{H}_{2} \mathrm{O}\right)_{2}$ cis

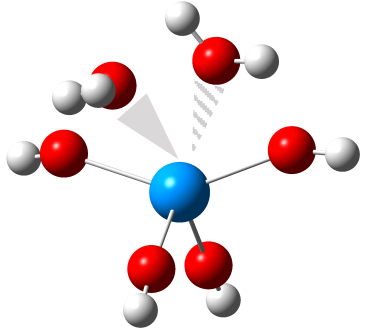

$\mathrm{U}(\mathrm{OH})_{4}\left(\mathrm{H}_{2} \mathrm{O}\right)_{2}$

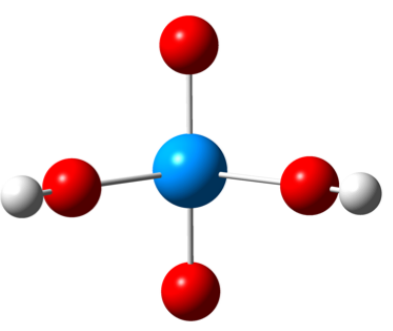

$\mathrm{UO}_{2}(\mathrm{OH})_{2}$

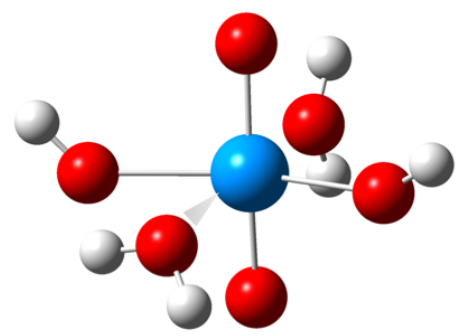

$\mathrm{UO}_{2}(\mathrm{OH})_{2}\left(\mathrm{H}_{2} \mathrm{O}\right)_{2}$ trans

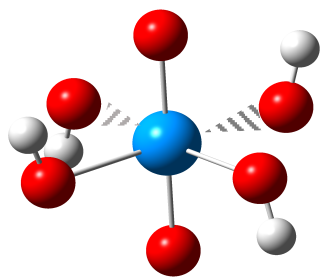

$\mathrm{UO}(\mathrm{OH})_{5}$

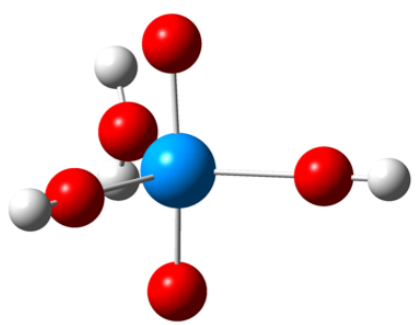

$\mathrm{UO}_{2}(\mathrm{OH})_{2}\left(\mathrm{H}_{2} \mathrm{O}\right)$

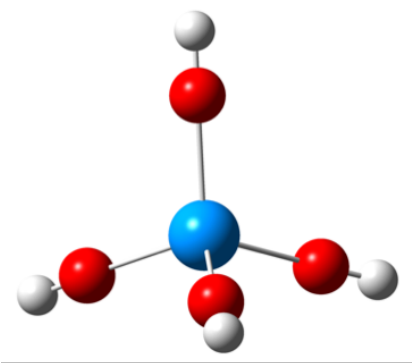

$\mathrm{U}(\mathrm{OH})_{4}$ 
Table S5. Molecules tested, corresponding to Table 1 in Main Text

\begin{tabular}{cc} 
molecule & In training set Y/N \\
\hline $\mathrm{UO}_{2} \mathrm{H}$ & Yes \\
$\mathrm{UH}_{3}$ & Yes \\
$\mathrm{UH}_{4}$ & Yes \\
$\mathrm{UO}_{2}^{2+}$ & Yes \\
$\mathrm{UO}_{2}\left(\mathrm{H}_{2} \mathrm{O}\right)^{2+}$ & Yes \\
$\mathrm{UO}_{2}\left(2 \mathrm{H}_{2} \mathrm{O}\right)^{2+}$ & Yes \\
$\mathrm{UO}_{2}\left(3 \mathrm{H}_{2} \mathrm{O}\right)^{2+}$ & Yes \\
$\mathrm{UO}_{2}\left(4 \mathrm{H}_{2} \mathrm{O}\right)^{2+}$ & Yes \\
$\mathrm{UO}_{2}\left(5 \mathrm{H}_{2} \mathrm{O}\right)^{2+}$ & Yes \\
$\mathrm{UO}_{2}(\mathrm{OH})^{+}$ & Yes \\
$\mathrm{UO}_{2}(\mathrm{OH})\left(\mathrm{H}_{2} \mathrm{O}\right)^{+}$ & Yes \\
$\mathrm{UO}_{2}(\mathrm{OH})\left(\mathrm{H}_{2} \mathrm{O}\right)_{2}{ }^{+}$ & Yes \\
$\mathrm{UO}_{2}(\mathrm{OH})\left(\mathrm{H}_{2} \mathrm{O}\right)_{3}{ }^{+}$ & Yes \\
$\mathrm{UO}_{2}(\mathrm{OH})_{2}$ & Yes \\
$\mathrm{UO}_{2}(\mathrm{OH})_{2}\left(\mathrm{H}_{2} \mathrm{O}\right)$ & Yes \\
$\mathrm{UO}_{2}(\mathrm{OH})_{2}\left(\mathrm{H}_{2} \mathrm{O}\right)_{2} \mathrm{cis}$ & Yes \\
$\mathrm{UO}_{2}(\mathrm{OH})_{2}\left(\mathrm{H}_{2} \mathrm{O}\right)_{2}$ trans & Yes \\
$\mathrm{U}(\mathrm{OH})_{4}$ & Yes \\
$\mathrm{H}^{-} \mathrm{U}_{-} \mathrm{O}$ & No \\
$\mathrm{UO} \mathrm{O}_{2}$ & No \\
$\mathrm{UO} \mathrm{O}_{3}$ & No \\
$\mathrm{UO}_{2}(\mathrm{OH})_{4}{ }^{2-}$ & No
\end{tabular}

Comparison of results for three molecules not in training set

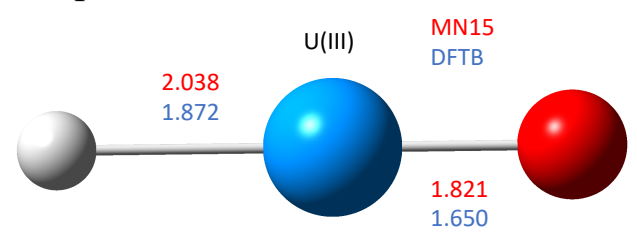

H-U-O has a multiconfigurational wave function with CI coefficients $\mathrm{c}_{1}=\mathrm{c}_{2}=0.69$

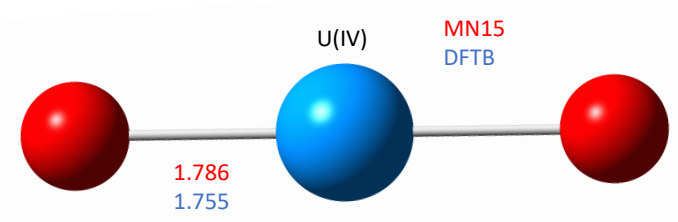




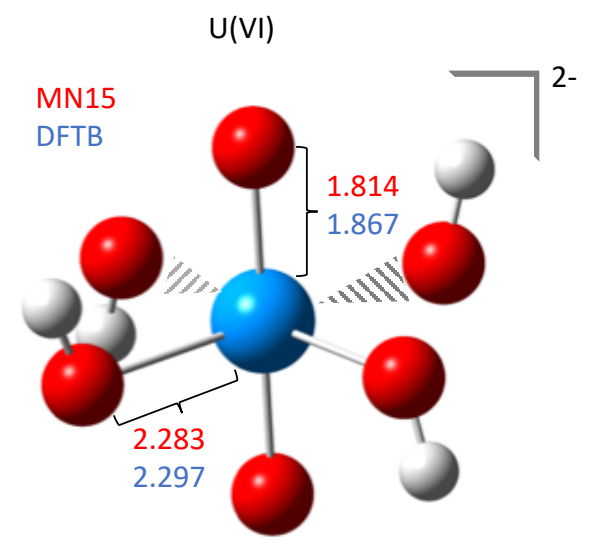


Table S6. Selected Geometries for CASPT2, MN15, and DFTB for $\mathrm{UO}_{2}{ }^{2+}$ and $\mathrm{UO}_{2}$

\begin{tabular}{cccc}
$\mathrm{UO}_{2}{ }^{2+}$ & $\mathrm{CAS}(12,12) / \mathrm{CASPT} 2$ & $\mathrm{MN} 15$ & $\mathrm{DFTB}$ \\
\hline $\mathrm{U}-\mathrm{O}$ (Ang) & 1.700 & 1.693 & 1.695 \\
$\mathrm{O}-\mathrm{U}-\mathrm{O}$ (degree) & 180.0 & 180.0 & 180.0 \\
\hline $\mathrm{UO}_{2}$ & & & 1.755 \\
$\mathrm{U}-\mathrm{O}$ (Ang) & 1.806 & 1.786 & 180.0
\end{tabular}

Table S7. Selected Geometries for CASPT2, MN15, and DFTB for $\mathrm{UO}_{2}(\mathrm{OH})^{+}$

\begin{tabular}{cccc} 
& CAS $(14,14) /$ CASPT2 & MN15 & DFTB \\
\hline U-O (Ang) & 1.743 & 1.734 & 1.737 \\
U-OH (Ang) & 1.996 & 1.990 & 2.012 \\
O-U-O (degrees) & 170.0 & 169.8 & 172.4
\end{tabular}

Table S8. Selected Geometries for CASPT2, MN15, and DFTB for $\mathrm{UO}_{2}\left(\mathrm{H}_{2} \mathrm{O}\right)^{2+}$

\begin{tabular}{cccc} 
& CAS $(14,14) /$ CASPT2 & MN15 & DFTB \\
\hline U-O (Ang) & 1.712 & 1.706 & 1.706 \\
U-OH (Ang) & 2.318 & 2.288 & 2.337 \\
O-U-O (degrees) & 178.6 & 177.2 & 177.6
\end{tabular}

Table S9. Selected Geometries for CASPT2, MN15, and DFTB for $\mathrm{UO}_{3}$

\begin{tabular}{cccc} 
& CAS $(12,13) /$ CASPT2 & MN15 & DFTB \\
\hline U-O (Ang) & 1.803 & 1.787 & 1.796 \\
U-O (Ang) & 1.841 & 1.834 & 1.844 \\
O-U-O (degrees) & 159.5 & 163.8 & 167.6 \\
O-U-O (degrees) & 100.3 & 98.1 & 96.2
\end{tabular}

Table S10. Selected Geometries for CASPT2, MN15, and DFTB for $\mathrm{UO}_{2} \mathrm{H}$

\begin{tabular}{cccc} 
& CAS $(9,11) /$ CASPT2 & MN15 & DFTB \\
\hline U-O (Ang) & 1.794 & 1.789 & 1.783 \\
U-H (Ang) & 2.041 & 2.015 & 2.067 \\
O-U-O (degrees) & 171.6 & 173.8 & 175.0 \\
H-U-O (degrees) & 94.2 & 93.1 & 92.5
\end{tabular}

Table S11. Selected Geometries for CASPT2, MN15, and DFTB for $\mathrm{UH}_{3}$

\begin{tabular}{llll} 
& CAS $(9,9) / C A S P T 2$ & MN15 & DFTB \\
\hline U-H (Avg) & 2.047 & 2.032 & 2.047
\end{tabular}


Table S12. Energy of Hydration (eV)

\begin{tabular}{llll} 
& DFT & DFTB & AE \\
\hline $\mathrm{UO}_{2}^{2+}+\mathrm{H}_{2} \mathrm{O} \rightarrow\left[\mathrm{UO}_{2}\left(\mathrm{H}_{2} \mathrm{O}\right)\right]^{2+}$ & -3.37 & -3.55 & 0.18 \\
$\mathrm{UO}_{2}{ }^{2+}+2 \mathrm{H}_{2} \mathrm{O} \rightarrow\left[\mathrm{UO}_{2}\left(\mathrm{H}_{2} \mathrm{O}\right)_{2}\right]^{2+}$ & -6.30 & -6.54 & 0.24 \\
$\mathrm{UO}_{2}^{2+}+3 \mathrm{H}_{2} \mathrm{O} \rightarrow\left[\mathrm{UO}_{2}\left(\mathrm{H}_{2} \mathrm{O}\right)_{3}\right]^{2+}$ & -8.84 & -9.07 & 0.23 \\
$\mathrm{UO}_{2}{ }^{2+}+4 \mathrm{H}_{2} \mathrm{O} \rightarrow\left[\mathrm{UO}_{2}\left(\mathrm{H}_{2} \mathrm{O}\right)_{4}\right]^{2+}$ & -10.94 & -11.1 & 0.16 \\
$\mathrm{UO}_{2}{ }^{2+}+5 \mathrm{H}_{2} \mathrm{O} \rightarrow\left[\mathrm{UO}_{2}\left(\mathrm{H}_{2} \mathrm{O}\right)_{5}\right]^{2+}$ & -12.33 & -12.0 & 0.33 \\
$\mathrm{MUE}$ & & & 0.23
\end{tabular}

Table S13. Stepwise Energy of Hydration (eV)

\begin{tabular}{llll} 
& DFT & DFTB & AE \\
\hline$\left[\mathrm{UO}_{2}\left(\mathrm{H}_{2} \mathrm{O}\right)\right]^{2+}+\mathrm{H}_{2} \mathrm{O} \rightarrow\left[\mathrm{UO}_{2}\left(\mathrm{H}_{2} \mathrm{O}\right)_{2}\right]^{2+}$ & -2.93 & -2.99 & 0.06 \\
{$\left[\mathrm{UO}_{2}\left(\mathrm{H}_{2} \mathrm{O}\right)_{2}\right]^{2+}+\mathrm{H}_{2} \mathrm{O} \rightarrow\left[\mathrm{UO}_{2}\left(\mathrm{H}_{2} \mathrm{O}\right)_{3}\right]^{2+}$} & -2.54 & -2.53 & 0.01 \\
{$\left[\mathrm{UO}_{2}\left(\mathrm{H}_{2} \mathrm{O}\right)_{3}\right]^{2+}+\mathrm{H}_{2} \mathrm{O} \rightarrow\left[\mathrm{UO}_{2}\left(\mathrm{H}_{2} \mathrm{O}\right)_{4}\right]^{2+}$} & -2.10 & -2.03 & 0.07 \\
{$\left[\mathrm{UO}_{2}\left(\mathrm{H}_{2} \mathrm{O}\right)_{4}\right]^{2+}+\mathrm{H}_{2} \mathrm{O} \rightarrow\left[\mathrm{UO}_{2}\left(\mathrm{H}_{2} \mathrm{O}\right)_{5}\right]^{2+}$} & -1.39 & -0.90 & 0.49 \\
$\mathrm{MUE}$ & & & 0.16
\end{tabular}

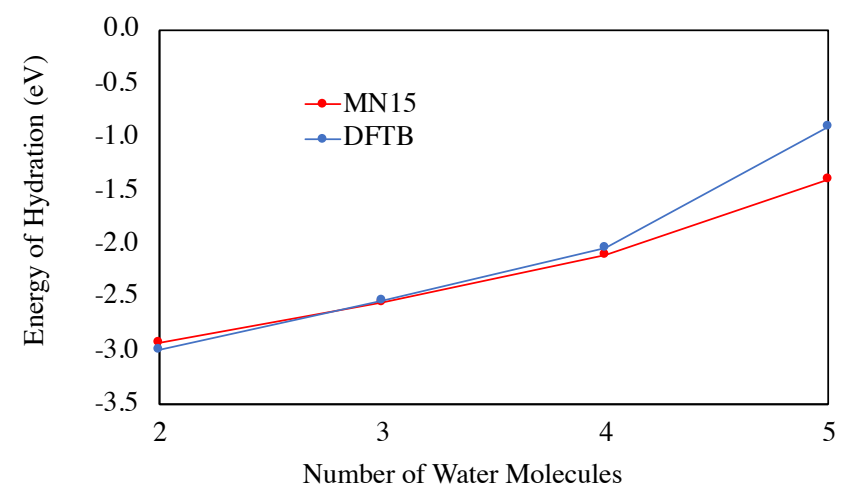

Figure S1. Stepwise energy of hydration of uranyl according to R2, where number of water molecules refers to the number of coordinating water molecules in the product of R2.

Table S14. Energy of Hydration (eV)

\begin{tabular}{llll} 
& DFT & DFTB & AE \\
\hline$\left[\mathrm{UO}_{2}(\mathrm{OH})\right]^{+}+\mathrm{H}_{2} \mathrm{O} \rightarrow\left[\mathrm{UO}_{2}(\mathrm{OH})\left(\mathrm{H}_{2} \mathrm{O}\right)\right]^{+}$ & -1.95 & -1.92 & 0.03 \\
{$\left[\mathrm{UO}_{2}(\mathrm{OH})\right]^{+}+2 \mathrm{H}_{2} \mathrm{O} \rightarrow\left[\mathrm{UO}_{2}(\mathrm{OH})\left(\mathrm{H}_{2} \mathrm{O}\right)_{2}\right]^{+}$} & -3.63 & -3.49 & 0.14 \\
{$\left[\mathrm{UO}_{2}(\mathrm{OH})\right]^{+}+3 \mathrm{H}_{2} \mathrm{O} \rightarrow\left[\mathrm{UO}_{2}(\mathrm{OH})\left(\mathrm{H}_{2} \mathrm{O}\right)_{3}\right]^{+}$} & -5.01 & -4.78 & 0.25 \\
{$\left[\mathrm{UO}_{2}(\mathrm{OH})\right]^{+}+4 \mathrm{H}_{2} \mathrm{O} \rightarrow\left[\mathrm{UO}_{2}(\mathrm{OH})\left(\mathrm{H}_{2} \mathrm{O}\right)_{4}\right]^{+}$} & -5.88 & -5.10 & 0.78 \\
$\mathrm{MUE}$ & & & 0.30
\end{tabular}


Table S15. Stepwise Energy of Hydration (eV)

\begin{tabular}{llll} 
& DFT & DFTB & AE \\
\hline$\left[\mathrm{UO}_{2}(\mathrm{OH})\left(\mathrm{H}_{2} \mathrm{O}\right)\right]^{+}+\mathrm{H}_{2} \mathrm{O} \rightarrow\left[\mathrm{UO}_{2}(\mathrm{OH})\left(\mathrm{H}_{2} \mathrm{O}\right)_{2}\right]^{+}$ & -1.68 & -1.57 & 0.11 \\
{$\left[\mathrm{UO}_{2}(\mathrm{OH})\left(\mathrm{H}_{2} \mathrm{O}\right)_{2}\right]^{+}+\mathrm{H}_{2} \mathrm{O} \rightarrow\left[\mathrm{UO}_{2}(\mathrm{OH})\left(\mathrm{H}_{2} \mathrm{O}\right)_{3}\right]^{+}$} & -1.38 & -1.27 & 0.11 \\
{$\left[\mathrm{UO}_{2}(\mathrm{OH})\left(\mathrm{H}_{2} \mathrm{O}\right)_{3}\right]^{+}+\mathrm{H}_{2} \mathrm{O} \rightarrow\left[\mathrm{UO}_{2}(\mathrm{OH})\left(\mathrm{H}_{2} \mathrm{O}\right)_{4}\right]^{+}$} & -0.87 & -0.34 & 0.53 \\
$\mathrm{MUE}$ & & & 0.25
\end{tabular}

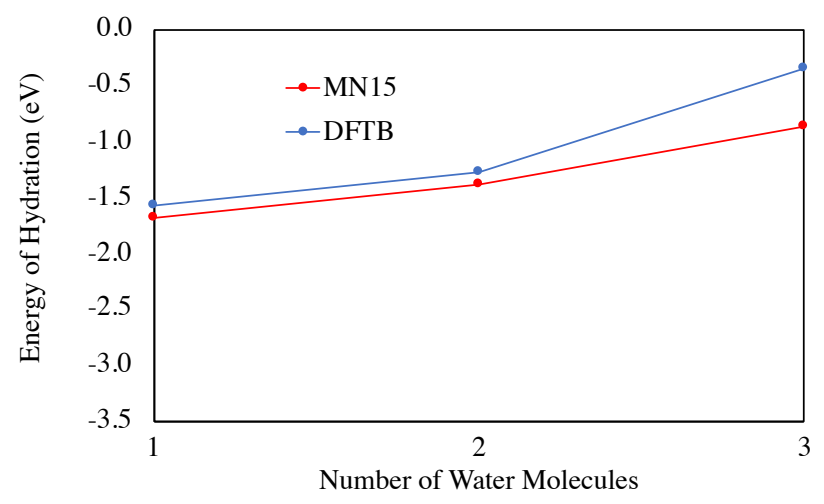

Figure S2. Stepwise energy of hydration of the $\left[\mathrm{UO}_{2}(\mathrm{OH})\left(\mathrm{H}_{2} \mathrm{O}\right)_{\mathrm{n}}\right]^{+}$unit, according to R4, where number of water molecules refers to the starting number of coordinating water molecules in the reactant side of $\mathrm{R} 4$.

Table S16. Energy of Hydrolysis (eV)

\begin{tabular}{llll} 
& DFT & DFTB & AE \\
\hline$\left[\mathrm{UO}_{2}\left(\mathrm{H}_{2} \mathrm{O}\right)_{4}\right]^{2+}+\mathrm{H}_{2} \mathrm{O} \rightarrow\left[\mathrm{UO}_{2}(\mathrm{OH})\left(\mathrm{H}_{2} \mathrm{O}\right)_{3}\right]^{+}$ & -1.77 & -2.30 & 0.54 \\
{$\left[\mathrm{UO}_{2}\left(\mathrm{H}_{2} \mathrm{O}\right)_{3}\right]^{2+}+\mathrm{H}_{2} \mathrm{O} \rightarrow\left[\mathrm{UO}_{2}(\mathrm{OH})\left(\mathrm{H}_{2} \mathrm{O}\right)_{2}\right]^{+}$} & -2.48 & -3.07 & 0.58 \\
{$\left[\mathrm{UO}_{2}\left(\mathrm{H}_{2} \mathrm{O}\right)_{2}\right]^{2+}+\mathrm{H}_{2} \mathrm{O} \rightarrow\left[\mathrm{UO}_{2}(\mathrm{OH})\left(\mathrm{H}_{2} \mathrm{O}\right)\right]^{+}$} & -3.35 & -4.02 & 0.67 \\
{$\left[\mathrm{UO}_{2}\left(\mathrm{H}_{2} \mathrm{O}\right)\right]^{2+}+\mathrm{H}_{2} \mathrm{O} \rightarrow\left[\mathrm{UO}_{2}(\mathrm{OH})\right]^{+}$} & -4.32 & -5.09 & 0.77 \\
MUE & & & 0.64
\end{tabular}




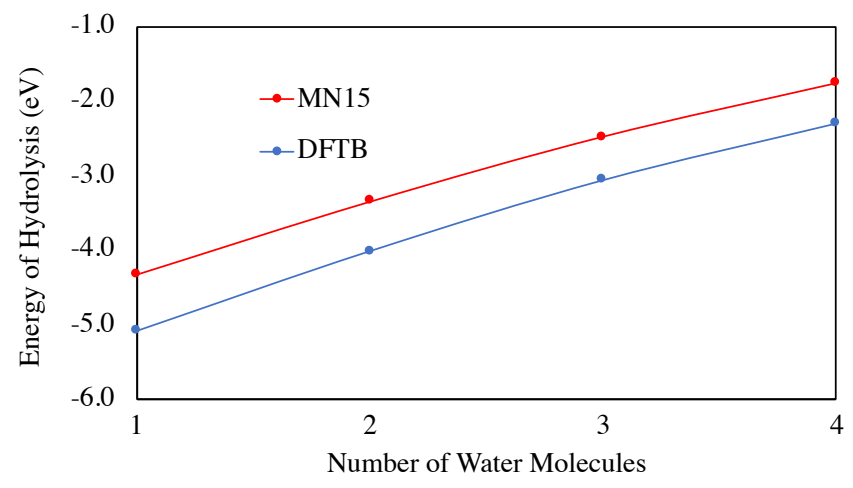

Figure S3. Energy of hydrolysis for R6, where number of molecules refers to the number of coordinating water molecules in the reactant side of R6.

Table S17. Energy of Dimerization (eV)

\begin{tabular}{llll} 
& DFT & DFTB & AE \\
\hline $2\left[\mathrm{UO}_{2}\left(\mathrm{H}_{2} \mathrm{O}\right)_{5}\right]^{2+} \rightarrow\left[\left(\mathrm{UO}_{2}\right)_{2}(\mathrm{OH})_{2}\left(\mathrm{H}_{2} \mathrm{O}\right)_{6}\right]^{2+}+2 \mathrm{H}_{3} \mathrm{O}^{+}$ & -0.43 & -0.67 & 0.24 \\
$2\left[\mathrm{UO}_{2}\left(\mathrm{H}_{2} \mathrm{O}\right)_{4}\right]^{2+}+2 \mathrm{H}_{2} \mathrm{O} \rightarrow\left[\left(\mathrm{UO}_{2}\right)_{2}(\mathrm{OH})_{2}\left(\mathrm{H}_{2} \mathrm{O}\right)_{6}\right]^{2+}+2 \mathrm{H}_{3} \mathrm{O}^{+}$ & -3.21 & -2.47 & 0.74 \\
$2\left[\mathrm{UO}_{2}\left(\mathrm{H}_{2} \mathrm{O}\right)_{3}\right]^{2+}+4 \mathrm{H}_{2} \mathrm{O} \rightarrow\left[\left(\mathrm{UO}_{2}\right)_{2}(\mathrm{OH})_{2}\left(\mathrm{H}_{2} \mathrm{O}\right)_{6}\right]^{2+}+2 \mathrm{H}_{3} \mathrm{O}^{+}$ & -7.41 & -6.53 & 0.87 \\
$2\left[\mathrm{UO}_{2}\left(\mathrm{H}_{2} \mathrm{O}\right)_{2}\right]^{2+}+6 \mathrm{H}_{2} \mathrm{O} \rightarrow\left[\left(\mathrm{UO}_{2}\right)_{2}(\mathrm{OH})_{2}\left(\mathrm{H}_{2} \mathrm{O}\right)_{6}\right]^{2+}+2 \mathrm{H}_{3} \mathrm{O}^{+}$ & -12.50 & -11.58 & 0.91 \\
$2\left[\mathrm{UO}_{2}\left(\mathrm{H}_{2} \mathrm{O}\right)\right]^{2+}+8 \mathrm{H}_{2} \mathrm{O} \rightarrow\left[\left(\mathrm{UO}_{2}\right)_{2}(\mathrm{OH})_{2}\left(\mathrm{H}_{2} \mathrm{O}\right)_{6}\right]^{2+}+2 \mathrm{H}_{3} \mathrm{O}^{+}$ & -18.35 & -17.57 & 0.78 \\
$\mathrm{MUE}$ & & & 0.71
\end{tabular}

Table S18. Energy of Dimerization (eV)

\begin{tabular}{llll} 
& DFT & DFTB & AE \\
\hline $2\left[\mathrm{UO}_{2}\left(\mathrm{H}_{2} \mathrm{O}\right)_{5}\right]^{2+}+\mathrm{H}_{2} \mathrm{O} \rightarrow\left[\left(\mathrm{UO}_{2}\right)_{2}(\mathrm{OH})(\mathrm{O})\left(\mathrm{H}_{2} \mathrm{O}\right)_{6}\right]^{+}+3 \mathrm{H}_{3} \mathrm{O}^{+}$ & -0.03 & -0.97 & 0.95 \\
$2\left[\mathrm{UO}_{2}\left(\mathrm{H}_{2} \mathrm{O}\right)_{4}\right]^{2+}+3 \mathrm{H}_{2} \mathrm{O} \rightarrow\left[\left(\mathrm{UO}_{2}\right)_{2}(\mathrm{OH})_{2}\left(\mathrm{H}_{2} \mathrm{O}\right)_{6}\right]^{2+}+3 \mathrm{H}_{3} \mathrm{O}^{+}$ & -2.81 & -2.77 & 0.03 \\
$2\left[\mathrm{UO}_{2}\left(\mathrm{H}_{2} \mathrm{O}\right)_{3}\right]^{2+}+5 \mathrm{H}_{2} \mathrm{O} \rightarrow\left[\left(\mathrm{UO}_{2}\right)_{2}(\mathrm{OH})_{2}\left(\mathrm{H}_{2} \mathrm{O}\right)_{6}\right]^{2+}+3 \mathrm{H}_{3} \mathrm{O}^{+}$ & -7.00 & -6.84 & 0.16 \\
$2\left[\mathrm{UO}_{2}\left(\mathrm{H}_{2} \mathrm{O}\right)_{2}\right]^{2+}+7 \mathrm{H}_{2} \mathrm{O} \rightarrow\left[\left(\mathrm{UO}_{2}\right)_{2}(\mathrm{OH})_{2}\left(\mathrm{H}_{2} \mathrm{O}\right)_{6}\right]^{2+}+3 \mathrm{H}_{3} \mathrm{O}^{+}$ & -12.09 & -11.88 & 0.21 \\
$2\left[\mathrm{UO}_{2}\left(\mathrm{H}_{2} \mathrm{O}\right)\right]^{2+}+9 \mathrm{H}_{2} \mathrm{O} \rightarrow\left[\left(\mathrm{UO}_{2}\right)_{2}(\mathrm{OH})_{2}\left(\mathrm{H}_{2} \mathrm{O}\right)_{6}\right]^{2+}+3 \mathrm{H}_{3} \mathrm{O}^{+}$ & -17.95 & -17.87 & 0.07 \\
$\mathrm{MUE}$ & & & 0.28
\end{tabular}

Table S19. Energy of Dimerization (eV)

\begin{tabular}{llll} 
& DFT & \multicolumn{1}{c}{ DFTB } & AE \\
\hline$\left[\mathrm{UO}_{2}\left(\mathrm{H}_{2} \mathrm{O}\right)_{5}\right]^{2+}+\left[\mathrm{UO}_{2}(\mathrm{OH})\left(\mathrm{H}_{2} \mathrm{O}\right)_{4}\right]^{+} \rightarrow\left[\left(\mathrm{UO}_{2}\right)_{2}(\mathrm{OH})(\mathrm{O})\left(\mathrm{H}_{2} \mathrm{O}\right)_{6}\right]^{+}+2 \mathrm{H}_{3} \mathrm{O}^{+}$ & 1.22 & 0.77 & 0.45 \\
{$\left[\mathrm{UO}_{2}\left(\mathrm{H}_{2} \mathrm{O}\right)_{5}\right]^{2+}+\left[\mathrm{UO}_{2}(\mathrm{OH})\left(\mathrm{H}_{2} \mathrm{O}\right)_{3}\right]^{+}+\mathrm{H}_{2} \mathrm{O} \rightarrow\left[\left(\mathrm{UO}_{2}\right)_{2}(\mathrm{OH})(\mathrm{O})\left(\mathrm{H}_{2} \mathrm{O}\right)_{6}\right]^{+}+2 \mathrm{H}_{3} \mathrm{O}^{+}$} & 0.35 & 0.43 & 0.08 \\
{$\left[\mathrm{UO}_{2}\left(\mathrm{H}_{2} \mathrm{O}\right)_{4}\right]^{2+}+\left[\mathrm{UO}_{2}(\mathrm{OH})\left(\mathrm{H}_{2} \mathrm{O}\right)_{2}\right]^{+}+3 \mathrm{H}_{2} \mathrm{O} \rightarrow\left[\left(\mathrm{UO}_{2}\right)_{2}(\mathrm{OH})(\mathrm{O})\left(\mathrm{H}_{2} \mathrm{O}\right)_{6}\right]^{+}+2 \mathrm{H}_{3} \mathrm{O}^{+}$} & -2.42 & -1.74 & 0.68 \\
{$\left[\mathrm{UO}_{2}\left(\mathrm{H}_{2} \mathrm{O}\right)_{3}\right]^{2+}+\left[\mathrm{UO}_{2}(\mathrm{OH})\left(\mathrm{H}_{2} \mathrm{O}\right)\right]^{+}+5 \mathrm{H}_{2} \mathrm{O} \rightarrow\left[\left(\mathrm{UO}_{2}\right)_{2}(\mathrm{OH})(\mathrm{O})\left(\mathrm{H}_{2} \mathrm{O}\right)_{6}\right]^{+}+2 \mathrm{H}_{3} \mathrm{O}^{+}$} & -6.20 & -5.34 & 0.85 \\
{$\left[\mathrm{UO}_{2}\left(\mathrm{H}_{2} \mathrm{O}\right)_{2}\right]^{2+}+\left[\mathrm{UO}_{2}(\mathrm{OH})\right]^{+}+7 \mathrm{H}_{2} \mathrm{O} \rightarrow\left[\left(\mathrm{UO}_{2}\right)_{2}(\mathrm{OH})(\mathrm{O})\left(\mathrm{H}_{2} \mathrm{O}\right)_{6}\right]^{+}+2 \mathrm{H}_{3} \mathrm{O}^{+}$} & -10.70 & -9.79 & 0.91 \\
$\mathrm{MUE}$ & & & 0.60
\end{tabular}


Table S20. Energy of Dimerization (eV)

\begin{tabular}{llll} 
& DFT & \multicolumn{1}{l}{ DFTB } & AE \\
\hline$\left[\mathrm{UO}_{2}\left(\mathrm{H}_{2} \mathrm{O}\right)_{5}\right]^{2+}+\left[\mathrm{UO}_{2}(\mathrm{OH})\left(\mathrm{H}_{2} \mathrm{O}\right)_{3}\right]^{+}+\mathrm{H}_{2} \mathrm{O} \rightarrow\left[\left(\mathrm{UO}_{2}\right)_{2}(\mathrm{OH})(\mathrm{O})\left(\mathrm{H}_{2} \mathrm{O}\right)_{6}\right]^{+}+2 \mathrm{H}_{3} \mathrm{O}^{+}$ & 0.35 & 0.43 & 0.08 \\
{$\left[\mathrm{UO}_{2}\left(\mathrm{H}_{2} \mathrm{O}\right)_{4}\right]^{2+}+\left[\mathrm{UO}_{2}(\mathrm{OH})\left(\mathrm{H}_{2} \mathrm{O}\right)_{3}\right]^{+}+2 \mathrm{H}_{2} \mathrm{O} \rightarrow\left[\left(\mathrm{UO}_{2}\right)_{2}(\mathrm{OH})(\mathrm{O})\left(\mathrm{H}_{2} \mathrm{O}\right)_{6}\right]^{+}+2 \mathrm{H}_{3} \mathrm{O}^{+}$} & -1.03 & -0.47 & 0.57 \\
{$\left[\mathrm{UO}_{2}\left(\mathrm{H}_{2} \mathrm{O}\right)_{3}\right]^{2+}+\left[\mathrm{UO}_{2}(\mathrm{OH})\left(\mathrm{H}_{2} \mathrm{O}\right)_{3}\right]^{+}+3 \mathrm{H}_{2} \mathrm{O} \rightarrow\left[\left(\mathrm{UO}_{2}\right)_{2}(\mathrm{OH})(\mathrm{O})\left(\mathrm{H}_{2} \mathrm{O}\right)_{6}\right]^{+}+2 \mathrm{H}_{3} \mathrm{O}^{+}$} & -3.14 & -2.50 & 0.63 \\
{$\left[\mathrm{UO}_{2}\left(\mathrm{H}_{2} \mathrm{O}\right)_{2}\right]^{2+}+\left[\mathrm{UO}_{2}(\mathrm{OH})\left(\mathrm{H}_{2} \mathrm{O}\right)_{3}\right]^{+}+4 \mathrm{H}_{2} \mathrm{O} \rightarrow\left[\left(\mathrm{UO}_{2}\right)_{2}(\mathrm{OH})(\mathrm{O})\left(\mathrm{H}_{2} \mathrm{O}\right)_{6}\right]^{+}+2 \mathrm{H}_{3} \mathrm{O}^{+}$} & -5.68 & -5.03 & 0.66 \\
{$\left[\mathrm{UO}_{2}\left(\mathrm{H}_{2} \mathrm{O}\right)_{1}\right]^{2+}+\left[\mathrm{UO}_{2}(\mathrm{OH})\left(\mathrm{H}_{2} \mathrm{O}\right)_{3}\right]^{+}+5 \mathrm{H}_{2} \mathrm{O} \rightarrow\left[\left(\mathrm{UO}_{2}\right)_{2}(\mathrm{OH})(\mathrm{O})\left(\mathrm{H}_{2} \mathrm{O}\right)_{6}\right]^{+}+2 \mathrm{H}_{3} \mathrm{O}^{+}$} & -8.61 & -8.02 & 0.59 \\
$\mathrm{MUE}$ & & & \\
\end{tabular}

Table S21. Energy of Dimerization (eV)

\begin{tabular}{llll} 
& DFT & \multicolumn{2}{c}{ DFTB } \\
\hline$\left[\mathrm{UO}_{2}\left(\mathrm{H}_{2} \mathrm{O}\right)_{5}\right]^{2+}+\left[\mathrm{UO}_{2}(\mathrm{OH})\left(\mathrm{H}_{2} \mathrm{O}\right)_{3}\right]^{+}+\mathrm{H}_{2} \mathrm{O} \rightarrow\left[\left(\mathrm{UO}_{2}\right)_{2}(\mathrm{OH})(\mathrm{O})\left(\mathrm{H}_{2} \mathrm{O}\right)_{6}\right]^{+}+2 \mathrm{H}_{3} \mathrm{O}^{+}$ & 0.35 & 0.43 & 0.08 \\
{$\left[\mathrm{UO}_{2}\left(\mathrm{H}_{2} \mathrm{O}\right)_{5}\right]^{2+}+\left[\mathrm{UO}_{2}(\mathrm{OH})\left(\mathrm{H}_{2} \mathrm{O}\right)_{2}\right]^{+}+2 \mathrm{H}_{2} \mathrm{O} \rightarrow\left[\left(\mathrm{UO}_{2}\right)_{2}(\mathrm{OH})(\mathrm{O})\left(\mathrm{H}_{2} \mathrm{O}\right)_{6}\right]^{+}+2 \mathrm{H}_{3} \mathrm{O}^{+}$} & -1.03 & -0.84 & 0.19 \\
{$\left[\mathrm{UO}_{2}\left(\mathrm{H}_{2} \mathrm{O}\right)_{5}\right]^{2+}+\left[\mathrm{UO}_{2}(\mathrm{OH})\left(\mathrm{H}_{2} \mathrm{O}\right)_{1}\right]^{+}+3 \mathrm{H}_{2} \mathrm{O} \rightarrow\left[\left(\mathrm{UO}_{2}\right)_{2}(\mathrm{OH})(\mathrm{O})\left(\mathrm{H}_{2} \mathrm{O}\right)_{6}\right]^{+}+2 \mathrm{H}_{3} \mathrm{O}^{+}$} & -2.71 & -2.41 & 0.30 \\
{$\left[\mathrm{UO}_{2}\left(\mathrm{H}_{2} \mathrm{O}\right)_{5}\right]^{2+}+\left[\mathrm{UO}_{2}(\mathrm{OH})\right]^{+}+4 \mathrm{H}_{2} \mathrm{O} \rightarrow\left[\left(\mathrm{UO}_{2}\right)_{2}(\mathrm{OH})(\mathrm{O})\left(\mathrm{H}_{2} \mathrm{O}\right)_{6}\right]^{+}+2 \mathrm{H}_{3} \mathrm{O}^{+}$} & -4.67 & -4.33 & 0.33 \\
MUE & & & 0.23
\end{tabular}

Table S22. Energy of Dimerization (eV)

\begin{tabular}{llll} 
& DFT & \multicolumn{1}{l}{ DFTB } & AE \\
\hline $2\left[\mathrm{UO}_{2}(\mathrm{OH})\left(\mathrm{H}_{2} \mathrm{O}\right)_{3}\right]^{+}+\mathrm{H}_{2} \mathrm{O} \rightarrow\left[\left(\mathrm{UO}_{2}\right)_{2}(\mathrm{OH})(\mathrm{O})\left(\mathrm{H}_{2} \mathrm{O}\right)_{6}\right]^{+}+\mathrm{H}_{3} \mathrm{O}^{+}$ & 0.73 & 1.83 & 1.11 \\
$2\left[\mathrm{UO}_{2}(\mathrm{OH})\left(\mathrm{H}_{2} \mathrm{O}\right)_{2}\right]^{+}+3 \mathrm{H}_{2} \mathrm{O} \rightarrow\left[\left(\mathrm{UO}_{2}\right)_{2}(\mathrm{OH})(\mathrm{O})\left(\mathrm{H}_{2} \mathrm{O}\right)_{6}\right]^{+}+\mathrm{H}_{3} \mathrm{O}^{+}$ & -2.04 & -0.71 & 1.33 \\
$2\left[\mathrm{UO}_{2}(\mathrm{OH})\left(\mathrm{H}_{2} \mathrm{O}\right)\right]^{+}+5 \mathrm{H}_{2} \mathrm{O} \rightarrow\left[\left(\mathrm{UO}_{2}\right)_{2}(\mathrm{OH})(\mathrm{O})\left(\mathrm{H}_{2} \mathrm{O}\right)_{6}\right]^{+}+\mathrm{H}_{3} \mathrm{O}^{+}$ & -5.39 & -3.85 & 1.55 \\
$2\left[\mathrm{UO}_{2}(\mathrm{OH})\right]^{+}+7 \mathrm{H}_{2} \mathrm{O} \rightarrow\left[\left(\mathrm{UO}_{2}\right)_{2}(\mathrm{OH})(\mathrm{O})\left(\mathrm{H}_{2} \mathrm{O}\right)_{6}\right]^{+}+\mathrm{H}_{3} \mathrm{O}^{+}$ & -9.31 & -7.69 & 1.61 \\
$\mathrm{MUE}$ & & & 1.40
\end{tabular}

Table S23. Energy of Dimerization (eV)

\begin{tabular}{llll} 
& DFT & DFTB & AE \\
\hline$\left[\mathrm{UO}_{2}\left(\mathrm{H}_{2} \mathrm{O}\right)_{5}\right]^{2+}+\left[\mathrm{UO}_{2}(\mathrm{OH})\left(\mathrm{H}_{2} \mathrm{O}\right)_{4}\right]^{+} \rightarrow\left[\left(\mathrm{UO}_{2}\right)_{2}(\mathrm{OH})(\mathrm{O})\left(\mathrm{H}_{2} \mathrm{O}\right)_{6}\right]^{+}+2 \mathrm{H}_{3} \mathrm{O}^{+}$ & 1.22 & 0.77 & 0.45 \\
{$\left[\mathrm{UO}_{2}\left(\mathrm{H}_{2} \mathrm{O}\right)_{4}\right]^{2+}+\left[\mathrm{UO}_{2}(\mathrm{OH})\left(\mathrm{H}_{2} \mathrm{O}\right)_{4}\right]^{+}+\mathrm{H}_{2} \mathrm{O} \rightarrow\left[\left(\mathrm{UO}_{2}\right)_{2}(\mathrm{OH})(\mathrm{O})\left(\mathrm{H}_{2} \mathrm{O}\right)_{6}\right]^{+}+2 \mathrm{H}_{3} \mathrm{O}^{+}$} & -0.17 & -0.13 & 0.04 \\
{$\left[\mathrm{UO}_{2}\left(\mathrm{H}_{2} \mathrm{O}\right)_{3}\right]^{2+}+\left[\mathrm{UO}_{2}(\mathrm{OH})\left(\mathrm{H}_{2} \mathrm{O}\right)_{4}\right]^{+}+2 \mathrm{H}_{2} \mathrm{O} \rightarrow\left[\left(\mathrm{UO}_{2}\right)_{2}(\mathrm{OH})(\mathrm{O})\left(\mathrm{H}_{2} \mathrm{O}\right)_{6}\right]^{+}+2 \mathrm{H}_{3} \mathrm{O}^{+}$} & -2.26 & -2.16 & 0.10 \\
{$\left[\mathrm{UO}_{2}\left(\mathrm{H}_{2} \mathrm{O}\right)_{2}\right]^{2+}+\left[\mathrm{UO}_{2}(\mathrm{OH})\left(\mathrm{H}_{2} \mathrm{O}\right)_{4}\right]^{+}+3 \mathrm{H}_{2} \mathrm{O} \rightarrow\left[\left(\mathrm{UO}_{2}\right)_{2}(\mathrm{OH})(\mathrm{O})\left(\mathrm{H}_{2} \mathrm{O}\right)_{6}\right]^{+}+2 \mathrm{H}_{3} \mathrm{O}^{+}$} & -4.81 & -4.69 & 0.12 \\
{$\left[\mathrm{UO}_{2}\left(\mathrm{H}_{2} \mathrm{O}\right)\right]^{2+}+\left[\mathrm{UO}_{2}(\mathrm{OH})\left(\mathrm{H}_{2} \mathrm{O}\right)_{4}\right]^{+}+4 \mathrm{H}_{2} \mathrm{O} \rightarrow\left[\left(\mathrm{UO}_{2}\right)_{2}(\mathrm{OH})(\mathrm{O})\left(\mathrm{H}_{2} \mathrm{O}\right)_{6}\right]^{+}+2 \mathrm{H}_{3} \mathrm{O}^{+}$} & -7.74 & -7.68 & 0.06 \\
$\mathrm{MUE}$ & & & 0.15
\end{tabular}




\section{Dimer Structures}

Table S24. Selected structural parameters (Angstrom) for $\left[\left(\mathrm{UO}_{2}\right)_{2}(\mathrm{OH})_{2}\left(\mathrm{H}_{2} \mathrm{O}\right)_{6}\right]^{2+}$

\begin{tabular}{|c|c|c|}
\hline & MN15 & DFTB \\
\hline U-U & 3.806 & 3.963 \\
\hline $\mathrm{U}-\mathrm{O}_{\mathrm{yl}}(\operatorname{avg})$ & 1.757 & 1.754 \\
\hline $\mathrm{U}-\mathrm{O}_{\mathrm{H}}$ & 2.318 & 2.381 \\
\hline $\mathrm{U}-\mathrm{H}_{2} \mathrm{O}$ (avg) & 2.494 & 2.493 \\
\hline
\end{tabular}

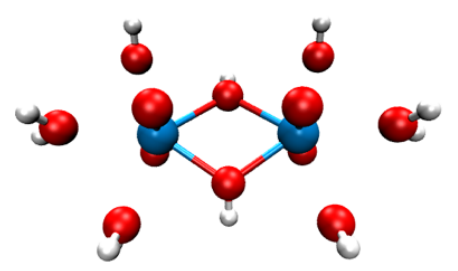

Fig S4. Structure of $\left[\left(\mathrm{UO}_{2}\right)_{2}(\mathrm{OH})_{2}\left(\mathrm{H}_{2} \mathrm{O}\right)_{6}\right]^{2+}$ where both DFTB and MN15 are qualitatively similar

Table S25. Selected structural parameters (Angstrom) for $\left[\left(\mathrm{UO}_{2}\right)_{2}(\mathrm{OH})(\mathrm{O})\left(\mathrm{H}_{2} \mathrm{O}\right)_{6}\right]^{+}$

\begin{tabular}{ccc} 
& MN15 & DFTB \\
\hline $\mathrm{U}-\mathrm{U}$ & 3.598 & 3.703 \\
$\mathrm{U}-\mathrm{O}_{\mathrm{yl}}(\mathrm{avg})$ & 1.773 & 1.776 \\
$\mathrm{U}-\mathrm{OH}$ & $2.318,2.437$ & 2.398 \\
$\mathrm{U}-\mathrm{O}$ & $2.155,2.115$ & 2.214 \\
$\mathrm{U}-\mathrm{H}_{2} \mathrm{O}($ avg $)$ & 2.534 & 2.539
\end{tabular}

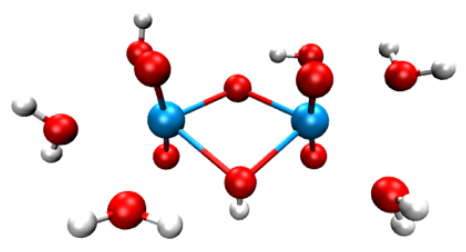

Fig. S5. Structure of $\left[\left(\mathrm{UO}_{2}\right)_{2}(\mathrm{OH})(\mathrm{O})\left(\mathrm{H}_{2} \mathrm{O}\right)_{6}\right]^{+}$at the MN15 level of theory, where two water molecules are hydrogen bonding with the bridging - $\mathrm{OH}$ and $-\mathrm{O}$ groups. These water molecules are farther way from $U$, compared to the other two on each side. 


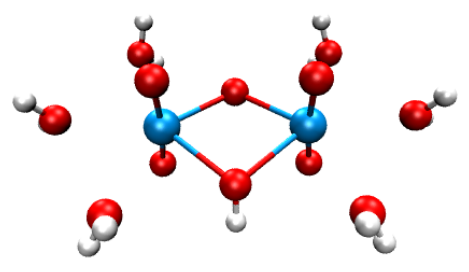

Fig. S6. Structure of $\left[\left(\mathrm{UO}_{2}\right)_{2}(\mathrm{OH})(\mathrm{O})\left(\mathrm{H}_{2} \mathrm{O}\right)_{6}\right]^{+}$at the DFTB level of theory

Table S26. Structural parameters for $\left[\left(\mathrm{UO}_{2}\right)_{2}(\mathrm{O})\left(\mathrm{H}_{2} \mathrm{O}\right)_{6}\right]^{2+}$ for selected bond lengths (Angstrom) and dihedral angles (degrees)

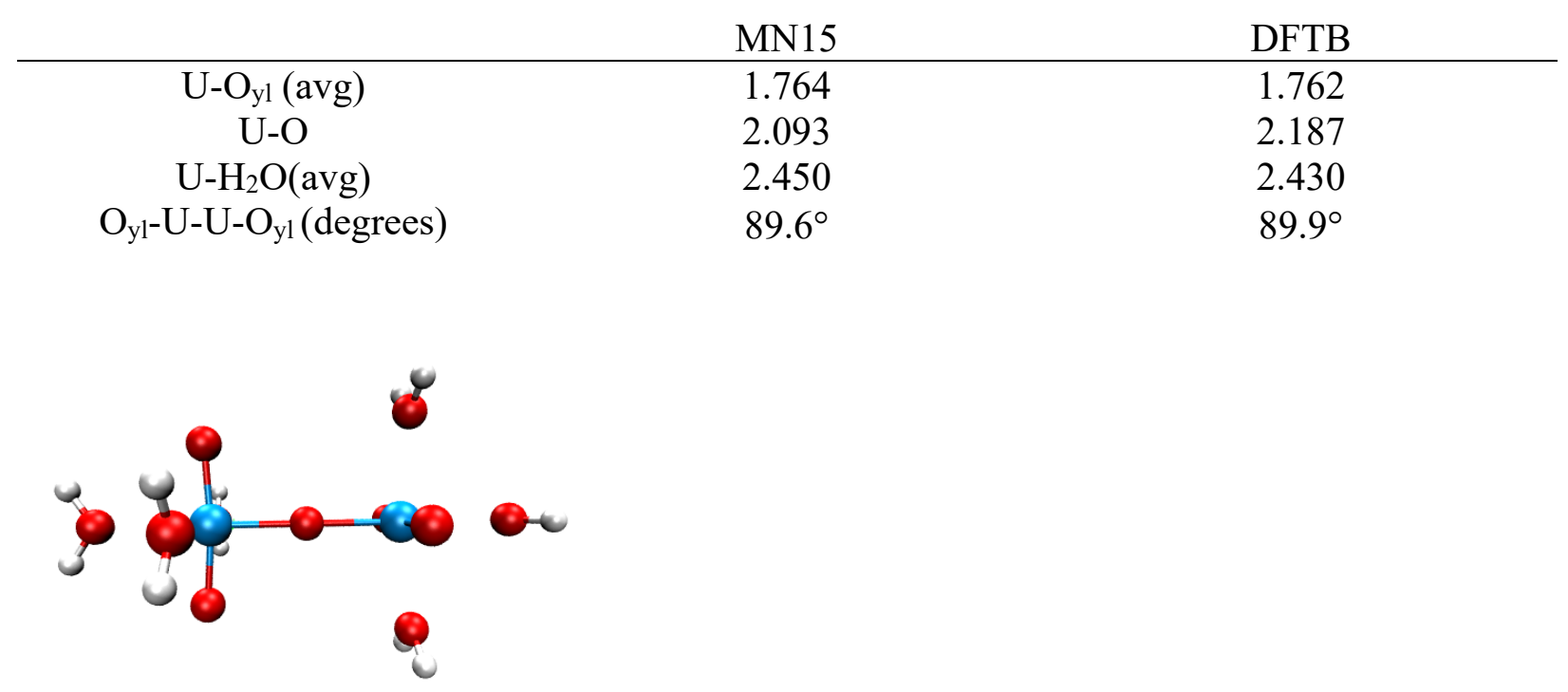

Fig. S7. Structure of $\left[\left(\mathrm{UO}_{2}\right)_{2}(\mathrm{O})\left(\mathrm{H}_{2} \mathrm{O}\right)_{6}\right]^{2+}$

Table S27. Structural parameters for $\left[\left(\mathrm{UO}_{2}\right)_{2}(\mathrm{OH})\left(\mathrm{H}_{2} \mathrm{O}\right)_{6}\right]^{3+}$ for selected bond lengths (Angstrom) and dihedral angles (degrees)

\begin{tabular}{ccc} 
& $\mathrm{MN} 15$ & DFTB \\
\hline $\mathrm{U}-\mathrm{O}_{\mathrm{yl}}$ & $1.751,1.741$ & $1.743,1.739$ \\
$\mathrm{U}-\mathrm{O}_{\mathrm{h}}$ & 2.329 & 2.412 \\
$\mathrm{U}-\mathrm{H}_{2} \mathrm{O}(\mathrm{avg})$ & 2.404 & 2.400 \\
$\mathrm{O}_{\mathrm{yl}}-\mathrm{U}-\mathrm{U}-\mathrm{O}_{\mathrm{yl}}$ (trans) & $117.1^{\circ}$ & $118.2^{\circ}$ \\
$\mathrm{U}-\mathrm{O}_{\mathrm{h}}-\mathrm{U}$ & $129.3^{\circ}$ & $150.8^{\circ}$
\end{tabular}




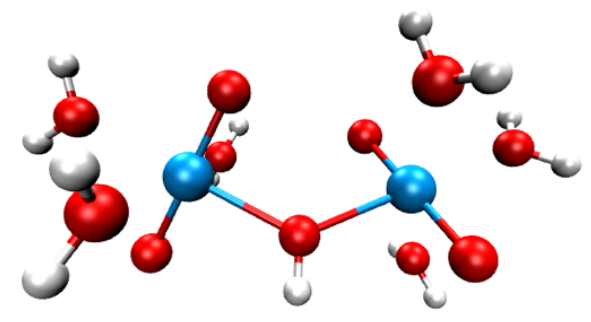

Fig. S8. Structure of $\left[\left(\mathrm{UO}_{2}\right)_{2}(\mathrm{OH})\left(\mathrm{H}_{2} \mathrm{O}\right)_{6}\right]^{3+}$ at the MN15 level of theory 